Method The University of Manchester in conjunction with the North West Paediatric Allergy Network developed and implemented a secure database utilising an online-system of live recording and collation of the workload and outcomes of children with allergies presenting to 21 paediatric secondary and tertiary centres across the whole North West of England and North Wales seeing children with allergies.

Results In a 9-month period from January to September 2019, 3,600 patient events were recorded across the two regions. $34 \%$ were new patients, $53 \%$ follow-up patients, 5\% telephone consultations and $8 \%$ did not attend their appointments. Eighty one percent of children had suffered immediate hypersensitivity reactions, of which $85 \%$ were children with food allergies. The 'big four' food allergies accounting for $81 \%$ of all children with food allergies were milk, egg, peanut and tree nuts. Only $19 \%$ of patients were discharged after their clinic appointment, while the remainder were followed up, 19\% of whom were put forward for a formal food challenge to confirm their allergies. Fourteen percent of children were either on oral immunosuppressants or biologics (1\%) or immunotherapy (oral immunotherapy for food allergies (9\%), subcutaneous or sublingual immunotherapy for aeroallergies (4\%)).

Conclusion Our online system for recording and collating of clinical workload and outcomes allows for immediate review of the number and types of allergies children suffered across the regions. Comparisons in terms of efficiency and outlies were easily detected. The data allowed us to develop business cases to meet current demands, as well as management pathways to streamline allergy management.

\section{G600(P) A CASE OF MYOCARDITIS DEVELOPING DESPITE TREATMENT FOR DRUG REACTION WITH EOSINOPHILIA AND SYSTEM SYMPTOMS (DRESS) SYNDROME}

${ }^{1} \mathrm{D}$ Denaxa, ${ }^{2} \mathrm{~S}$ Hughes, ${ }^{3} \mathrm{~A}$ Chieng, ${ }^{4} \mathrm{G}$ Vassalo, ${ }^{5}$ Kammaly, ${ }^{5} \mathrm{E}$ Lawson, ${ }^{6} \mathrm{D}$ Naisbitt. ${ }^{1}$ Paediatric Allergy and Immunology, Royal Manchester Children's Hospital, Manchester, UK; ${ }^{2}$ Paediatric Allergy and Immunology, University of Manchester, Royal Manchester Children's Hospital, Manchester, UK; ${ }^{3}$ Paediatric Rheumatology, Royal Manchester Children's Hospital, Manchester, UK; ${ }^{4}$ Paediatric Neurology, Royal Manchester Children's Hospital, Manchester, UK; ${ }^{5}$ Paediatric Neurosurgery, Royal Manchester Children's Hospital, Manchester, UK; ${ }^{6}$ Molecular and Clinical Pharmacology, University of Liverpool, Liverpool, UK

\subsection{6/archdischild-2020-rcpch.515}

Aim We present a case of an eight year old girl who was treated with intravenous antibiotics for group A streptococcus (GAS) peritonitis. She subsequently became severely unwell with skin rash and multiorgan dysfunction syndrome. A rare complication and unusual progress are described.

Results The patient had a background of ventriculoperitoneal (VP) shunt for hydrocephalus at five months of age. She was transferred in mid March 2019 to the tertiary hospital with abdominal sepsis. In the first two weeks she had surgical and neurosurgical interventions on three occasions. Chains of gram positive cocci were seen in peritoneal fluid and GAS was identified from cerebrospinal fluid. Treatment was ceftriaxone and metronidazole.

Five weeks later she developed a morbilliform rash, fever, eosinophilia, renal, liver impairment and lactic acidosis. She was admitted to PICU, requiring intubation and multiorgan support. The working diagnosis was DRESS syndrome using the Japanese consensus group diagnostic criteria. Stopping antibiotics was advised, but instead they were changed (to meropenem and vancomycin), and she was treated with steroids. She clinically improved shortly before having a further significant clinical deterioration with myocarditis. Her atrial natriuretic peptide was more than $70,000 \mathrm{pg} / \mathrm{ml}$ and troponin-T raised at a level of $243 \mathrm{ng} / \mathrm{L}$. Because myocarditis was not expected, a wider differential was considered including sepsis and rheumatic fever (revised Jones criteria met: rising GAS titre, carditis, rash, fever, high inflammatory markers). Although American College for Rheumatology criteria not fully met as did not have tissue biopsy, eosinophilic granulomatosis with polyangitis was also considered (criteria met: eosinophilia, lung infiltrates on CT of chest, neuropathy ). She improved with milrinone, pulsed high dose methyl-prednisolone, cyclophosphamide and plasma exchange.

Later, lymphocyte transformation assays showed responses to ceftriaxone and vancomycin, but not to other agents.

Conclusions This is a rare case where DRESS myocarditis developed despite appropriate medication change and treatment with steroids. Clinical multidisciplinary team decision making was key to securing an optimal outcome.

\section{G601(P) ABSTRACT WITHDRAWN}

\section{G602(P) IS HUMAN CHALLENGE AN ACCEPTABLE METHODOLOGY IN PREGNANCY: AN INTERVIEW STUDY}

${ }^{1}$ RB Dorey, ${ }^{2} \mathrm{AA}$ Theodosiou, ${ }^{3} \mathrm{TF}$ Vandrevala, ${ }^{2} \mathrm{RC}$ Read, ${ }^{4} \mathrm{CE}$ Jones. ${ }^{1} \mathrm{~N} I H R$ Southampton Clinical Research Facility, University of Southampton, Southampton, UK; ${ }^{2}$ Faculty of Medicine, University of Southampton, Southampton, UK; ${ }^{3}$ Department of Psychology, Kingston University, London, UK; ${ }^{4}$ Clinical and Experimental Sciences, University of Southampton, Southampton, UK

\subsection{6/archdischild-2020-rcpch.516}

Introduction Human challenge studies are becoming increasingly common as a method for investigating vaccines and the relationships between commensal microbes and humans. To date, these studies have been conducted exclusively in healthy adult populations. There is a significant burden from infectious diseases in the pregnant and neonatal population. Human challenge studies in pregnancy may help us elucidate the mechanisms underpinning risk from microbial transfer from mother to infant and help with vaccine development. However, it is unclear whether these studies would be acceptable to pregnant women. Therefore, we undertook an interview study to investigate whether human challenge studies would be acceptable to a pregnant population.

Methods We recruited healthy pregnant women from a maternity hospital until data saturation was reached. We invited them to take part in an interview study that explored perception of risk and acceptability of a hypothetical human challenge study in pregnancy. We conducted qualitative analysis of interview transcripts to identify themes and patterns present.

Results A significant proportion of the participants interviewed found a human challenge study in pregnancy to be acceptable. They reported that their participation in such a study could be encouraged by detailed explanations of risk and how study design would mitigate these. They reported that a degree of uncertainty would not dissuade them from participating. We found that participants were polarised in their opinions of this hypothetical study and early in the interview formed opinions about acceptability. Finally, once these opinions were formed 
reassurance did not change participants initial impressions of acceptability.

Conclusion Conducting a human challenge study in pregnancy would be acceptable to some pregnant women and may offer a means to investigate the specific environment that surrounds commensalisation of neonates. Furthermore, this may offer new avenues to explore vaccine development for the prevention of neonatal infection. While participants would want the maximum amount of information available before considering whether to participate, initial aversion to this methodology it is unlikely to be changed.

\section{G603(P) NEONATAL CHIKUNGUNYA: AN INDIAN PERSPECTIVE}

R Shriyan. Dept of Paediatrics, Vardhman Mahavir Medical College and Safdarjung Hospital, New Delhi, India

\subsection{6/archdischild-2020-rcpch.517}

Introduction Chikungunya is neonates is a rare entity with varied clinical presentation and poses a diagnostic challenge in neonates.

Methods Four neonates were studied during the period of June to September 2016, admitted to the NICU,born to symptomatic mothers and IgM serology positive. Each of the babies was enrolled after a written informed consent. In addition to a thorough clinical history and examination these babies underwent a sepsis screen, blood culture, Chikungunya IgM ELISA and CSF examination.

Results

\begin{tabular}{|c|c|c|c|c|}
\hline Profile & Case 1 & Case 2 & Case 3 & Case 4 \\
\hline $\begin{array}{l}\text { Maternal history- } \\
\text { fever, rash, athralgia }\end{array}$ & $\begin{array}{l}2 \text { days prior to } \\
\text { delivery }\end{array}$ & $\begin{array}{l}4 \text { days prior } \\
\text { to delivery }\end{array}$ & $\begin{array}{l}7 \text { days prior } \\
\text { to delivery }\end{array}$ & $\begin{array}{l}5 \text { days prior } \\
\text { to delivery }\end{array}$ \\
\hline $\begin{array}{l}\text { Maternal } \\
\text { Chikungunya } \\
\text { serology IgM } \\
\text { Signs and Symptoms }\end{array}$ & Positive & Positive & Positive & Positive \\
\hline Fever & Present & Present & Present & Present \\
\hline Skin rash & Present & Present & Present & Present \\
\hline Refusal to feed & Present & Present & Present & Present \\
\hline Apnea & Absent & Present & Absent & Present \\
\hline Seizures & Present & Present & Absent & Present \\
\hline Shock & Absent & Present & Absent & Present \\
\hline $\begin{array}{l}\text { Encephalopathy } \\
\text { Investigations }\end{array}$ & Present & Present & Absent & Present \\
\hline Sepsis screen & Negative & Negative & Negative & Negative \\
\hline CRP & Present & Present & Present & Present \\
\hline Platelet count & 30,000/cu.mm & $\begin{array}{l}1,25,000 / \mathrm{cu} . \\
\mathrm{mm}\end{array}$ & $\begin{array}{l}1,57,000 / \mathrm{cu} . \\
\mathrm{mm}\end{array}$ & $\begin{array}{l}1,00,000 / \mathrm{cu} . \\
\mathrm{mm}\end{array}$ \\
\hline CSF & Hypoglycorrhagia & Normal & Normal & Normal \\
\hline $\begin{array}{l}\text { Chikungunya serology } \\
\text { IgM }\end{array}$ & Positive & Positive & Positive & Positive \\
\hline Ventilatory support & Required ( 2 days) & $\begin{array}{l}\text { Required ( } 2 \\
\text { days) }\end{array}$ & Not required & $\begin{array}{l}\text { Required } \\
\text { (3 days) }\end{array}$ \\
\hline Outcome & Discharge & Discharge & Discharge & Discharge \\
\hline
\end{tabular}

Conclusion A strong clinical suspicion should be kept when a newborn presents with features of encephalopathy, perioral hyperpigmentation especially in endemic areas when the mother has been viremic in the pre and intrapartum period.

\section{G604(P) FIVE CASES OF LYME DISEASE PRESENTING WITHIN A SPAN OF THREE DAYS}

O Oladiran, B Rai, P Sundaram, S bandi. Paediatrics, Leicester Royal Infirmary, Leicester, UK

\subsection{6/archdischild-2020-rcpch.518}

Background Incidence of Lyme disease in UK has been estimated as 3000 per year by Public Health England, and recent observations have raised doubt about its true incidence as it is not always reported and sometimes can undergo undiagnosed in view of its presentation as flu like illness. It is caused by Borrelia, a spirochete bacteria, transmitted by the bite of infected ticks.

Case Series Case 1: A four year old boy presented on 9th days after he had multiple insect bites while out on a boat. Initially he had developed macular rashes with mild flu like symptoms which resolved spontaneously. Few of his rashes kept enlarging and became target like lesions before he visited emergency department. Examination revealed typical target lesions over left deltoid area. Systemic examination including neurological examination were normal. He was allergic to penicillin and henceforth treated with Azithromycin for 17 days. As his presentation was typical, no investigations were required.

Case 2: A seven year old boy presented to ED with six erythema migrans rash, a day after he was bitten by insects in a park. He developed rash within a day of tick bite. He was systemically well. As he gave a distinctive history of tick bite and rashes were characteristics of the Lyme disease, no investigations were required. He was treated with Azithromycin and recovered well.

Case 3: A twelve year old female presented with erythema migrans rash two days after she was being bitten by ticks in the same park as the patient in case 2. She had no headache or cardiac symptoms. She was treated with Doxycycline for 21 days (as per NICE recommendations) and she recovered well.

Case 4 and 5: Presentations were similar to case 3 with characteristics rash and responded well to treatment.

Conclusions Characteristics rash in Lyme disease showed wide range of its first notifiable change (enlargement with central clearing and appearance of target like lesions) in our cohort of cases. It ranged from as early as 24 hours up to 9 days. History is vital in arriving at initial diagnosis.

\section{G605(P) THE MISSING LINK IN PREVENTING TETANUS DEATHS IN NIGERIAN CHILDREN}

${ }^{1} \mathrm{OM}$ Awofeso, ${ }^{2} \mathrm{Y}$ Shittu, ${ }^{2} \mathrm{C}$ Akinde, ${ }^{3} \mathrm{AA}$ Roberts, ${ }^{2} \mathrm{O}$ Buchi-Njere. ${ }^{1}$ Department of Paediatrics, Lagos University Teaching Hospital, Lagos, Nigeria; ${ }^{2}$ Department of Research, The Nigerian Child Initiative, Lagos, Nigeria; ${ }^{3}$ Department of Community Health and Primary Care, College of Medicine, University of Lagos, Lagos, Nigeria

\subsection{6/archdischild-2020-rcpch.519}

Background Global evidence reports tetanus as one of the highest contributors to preventable child deaths. Despite increasing advances in medicine, Nigeria still contributes a large burden to unnecessary child deaths having failed to eradicate tetanus.

Methods A multipronged cross-sectional study aimed at identifying contributors to tetanus deaths in children in Lagos state. A 5-year retrospective hospital survey was carried in a tertiary centre to identify paediatric admissions due to tetanus, 